\title{
Development of a Dedicated Precision Polarimeter for Charged Particle EDM searches at COSY
}

\author{
I. Keshelashvili*1, O. Javakhishvili ${ }^{1,2}$, D. Mchedlishvili ${ }^{3,4}$, D. Shergelashvili ${ }^{4}$ \\ (on behalf of the JEDI collaboration) \\ ${ }^{1}$ Institut für Kernphysik,, Forschungszentrum Jülich, 52425 Jülich, Germany \\ ${ }^{2}$ Department of Electrical and Computer Engineering, Agricultural University of Georgia, 0159 \\ Tbilisi, Georgia \\ ${ }^{3}$ High-Energy Physics Institute, Tbilisi State University, 0186 Tbilisi, Georgia \\ ${ }^{4}$ Tbilisi State University, SMART|EDM Laboratory, 0179 Tbilisi, Georgia \\ E-mail: i.keshelashvili@fz-juelich.de
}

\begin{abstract}
The international JEDI (Jülich Electric Dipole moment Investigation) collaboration is preparing a first-ever direct measurement of the deuteron Electric Dipole Moment (EDM), using the COSY storage ring at Forschungszentrum Jülich (Germany). A new polarimeter is required to detect the very slow and minuscule polarization change with time: starting in 2016, we have designed, built and commissioned a new modular polarimeter based on LYSO inorganic scintillator crystals. The polarimeter concept exploits LYSO modules $\left(3 \times 3 \times 8 \mathrm{~cm}^{3}\right)$, individually coupled to modern large area SiPM arrays which are operating at low voltage compared to conventional vacuum photo tubes. The detector system and its vacuum system have radial symmetry and a thin exit window, making the polarimeter very efficient for online up-down and left-right asymmetry measurements. After several tests at the external COSY beam, we have recently installed the complete system in the COSY ring for use with internal circulating beams. We have performed two commissioning beam times to test the detector at various polarized beam conditions. Now it is ready to be employed as the general purpose polarimeter for JEDI and possibly other users at COSY.
\end{abstract}

The 18th International Workshop on Polarized Sources, Targets, and Polarimetry, PSTP2019

23-27 September, 2019

Knoxville, Tennessee

*Speaker. 


\section{Introduction}

The search for an electric dipole moment (EDM) aligned along the spin axis of an elementary particle is of fundamental importance [1]. Since this configuration violates both parity $(\mathrm{P})$ and time-reversal $(\mathrm{T})$ invariance, its observation at presently achieved or in future available sensitivity levels may point to a potential new source of CP-violation. Such a violation is needed to explain the dominance of matter over anti-matter in our post-Big-Bang universe [2]. Such searches have been conducted on neutrons confined in traps. Further EDM limits on elementary particles have been obtained for the muon, tauon and moderately for the electron. For the proton and deuteron, there are no direct measurements up to date. The JEDI collaboration [3] is planning to accomplish a first direct measurement of deuteron EDM. This measurement will take place at the existing accelerator facility COSY [4] at Forschungszentrum Jülich using a newly build polarimeter, see Fig.: 1. The

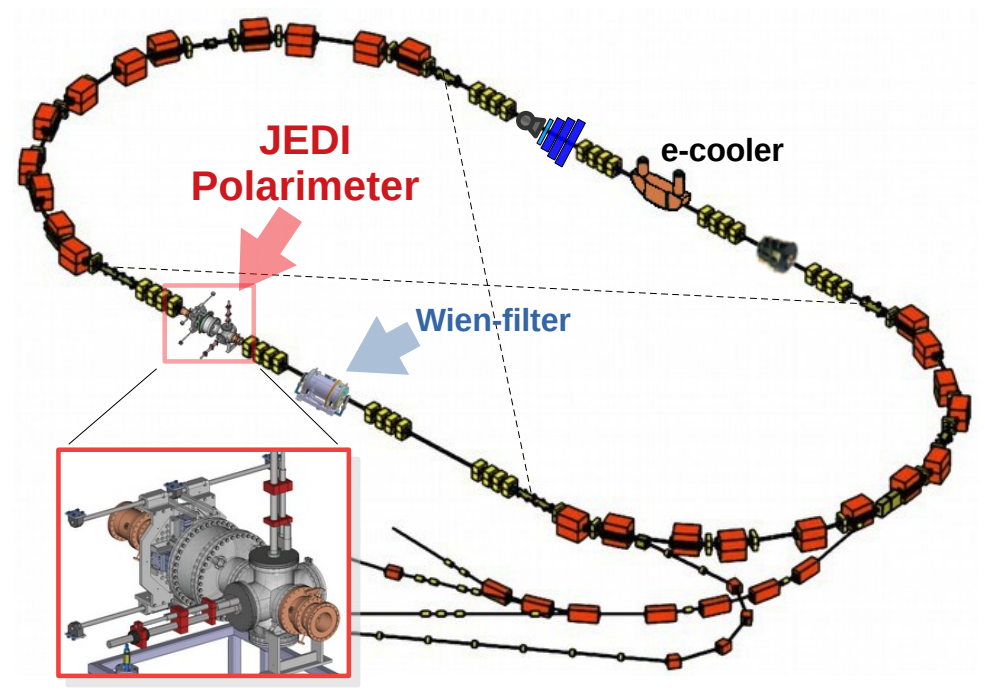

Figure 1: The COSY accelerator and storage ring with the necessary equipment for EDM search. The new polarimeter is shown as an insert.

method of choice at COSY is the so-called "partially frozen spin" [5] by using an RF Wien filter [6], which was recently installed at COSY and commissioned successfully. In the past years, we have demonstrated the requested long (more than $1000 \mathrm{~s}$ ) spin coherency times after polarization vector is rotated in horizontal plane $[7,8,9]$.

\section{Polarimeter and its operation}

The requirements of a polarimeter for an EDM search are foremost a high efficiency. Here, we define efficiency as a ratio between detected elastic events and extracted particles from the beam. We need to be able to sample as many of the beam particles as possible and derive a very high-statistics measurement of an asymmetry the signal for an EDM. With that goes high analyzing power and a large cross-section for elastic scattering [10]. The polarimeter must identify elastic events by a precision $\Delta \mathrm{E}$ vs $\mathrm{E}$ measurement technique. The total energy must be extracted without 
a magnetic spectrometer, which brings us to the natural choice of a scintillating calorimeter [11, 12]. We selected the novel inorganic LYSO material for its superior properties. High light output resulting in high resolution, short light emission decay time resulting in a fast signal, a very high melting point resulting best radiation hardness. To avoid stray fields from the high voltage supply for PMTs, modern large area silicon photomultipliers (SiPM) have been tested[13, 14] and found superior compared to conventional PMTs. The SiPM supply voltage can be decreased down to 27 $\mathrm{V}$ or lower which is clear advantage against PMTs which are mostly supplied in $\mathrm{kV}$ ranges [15]. SiPMs are also more compact and deliver excellent energy resolution [16].

In the first tests at COSY, the question was how to optimize a target and detector scheme that would deliver the desired efficiency. What has been started and has been used ever since is a scheme in which the beam is placed close to the block target (usually about $1 \mathrm{~mm}$ away from the nearest flat face). Then the beam is excited by putting narrow band around the betatron frequency on a set of stripline plates (two plates that create an electric field across the beam) and feeding in frequencies that are close to the resonance or its harmonics. That makes particles in the beam oscillate in the quadrupole focusing fields of the storage ring smeared by the one $\mathrm{kHz}$ white noise. For our setup, there are usually about 3.6 oscillations (the beam tune) per turn, and shortly after that, the particle hits the front of the block some distance (typically $0.2 \mathrm{~mm}$ ) in from the edge. Now it goes through the whole target material, thus having the chance to hit a nucleus and undergo larger-angle scattering into the polarimeter.

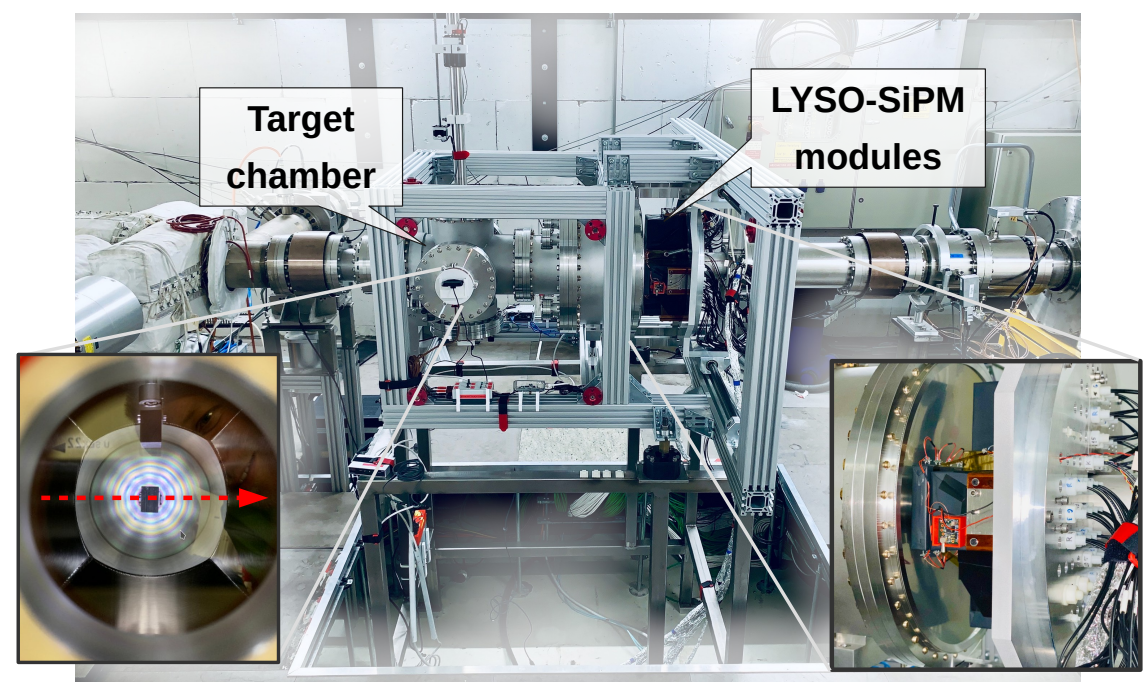

Figure 2: JEDI polarimeter installed in the COSY ring. Left insert: view of the target chamber where vertical and horizontal graphite block target are shown. Right insert: the installed LYSO-SiPM modules with the plastic scintillator $\Delta E$ layer is depicted.

\section{Installation in the COSY ring}

In summer 2019, the polarimeter consisting of 52 LYSO-SiPM modules and front plastic scintillator $\Delta E$ counters had been installed in the COSY ring. Figure 2 shows the final installation with $2 \mathrm{~cm}$ thick graphite block targets in vertical and horizontal direction. Before the installation, 
the vacuum system has been tested in the laboratory setup reaching $10^{-12}$ mbar pressure, which fulfills COSY UHV specifications. Excellent vacuum conditions have been achieved by using a fully isolated magnetic feed-through of the target system. In the future upgrades, Rogowsky beam positioning coils [17] will be added at the entrance and exit flanges of the polarimeter to monitor the beam crossing trough the detector. This will improve our control of systematic errors for the asymmetry measurement. The voltage supply of the SiPM and the VME based SADC [18] readout systems are installed outside of the accelerator tunnel. In addition, five digital temperature monitoring IC's are distributed over modules, tunnel, and voltage supply box. All systems can be controlled remotely from the experimental control room. The target movement is also remotely controlled allowing fast adaptation and modifications for the different experimental conditions. Two web cameras are also added for live monitoring purposes. The whole detector has been operated during two commissioning beam times and demonstrated its stability.
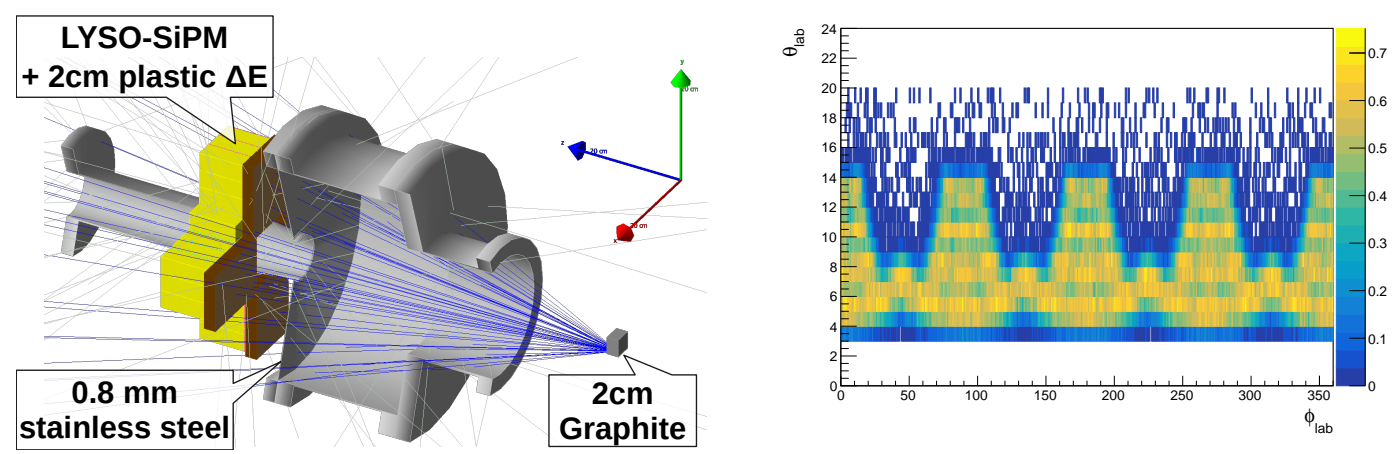

Figure 3: Left: Geant4 model of the polarimeter with a carbon target and LYSO modules shadowed by plastic scintillators. Right: The angular acceptance and the detection efficiency calculated by the detected to generated event ratio for deuteron carbon scattering at $230 \mathrm{MeV}$. $180 \mathrm{MeV}$ energy cut in LYSO energy is used to select elastic scattering events.

\section{Results}

In parallel to hardware and readout software development, the Monte-Carlo simulation code based on the CERN Geant4 library has been developed. The detector components are fully described in the code (see Fig. 3). The output can be analyzed similarly to the experimental data. Simulated energy losses can be used for detector calibration and systematic error estimations. We have done a series of studies to estimate artificial asymmetries caused by vertical and horizontal block targets. Also, the displacement and rotation of the initial scattering have been investigated carefully. It should be noted that all detector-caused asymmetries are constant and can be easily distinguished from the precessing polarization asymmetry, which is the case during our experiments.

Figure 4 shows measurements of the in-plain polarization (IPP) as a function of time after the polarization was rotated into the horizontal plane at $100 \mathrm{~s}$. The measurements were made with one beam polarization state. After the very first second, which is spent in the COSY cycle to inject and accelerate the deuteron beam up to the desired $230 \mathrm{MeV}$ energy, 50 s electron cooling is applied. In the next step, we measure vertical polarization, which represents here zero horizontal polarization. 


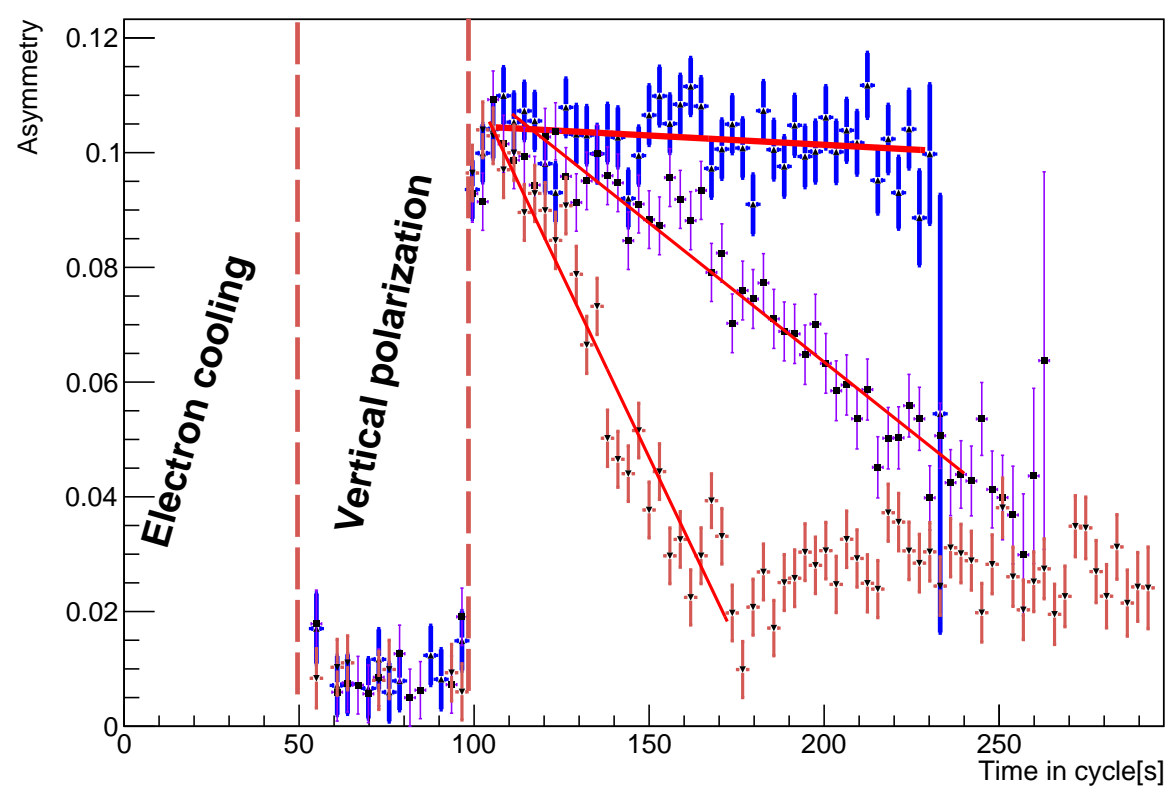

Figure 4: Horizontal axis shows a time within the COSY cycle vs. oscillating up-down asymmetry caused by horizontally precessing polarization vector. Three different spin coherent times are shown, each for different chromatisity settings corresponding to various spin coherence times.

After that, the polarization vector is rotated into the horizontal plane using RF solenoid. The three linear fit functions represent three different sextupole magnet settings, resulting in polarization lifetimes, defined as the time for the normalized IPP to reach respectively.

\section{Summary}

To conclude, during the development period, we have determined the module assembly procedures in a fast and efficient way. Two assembly stations have been set up to make fast fabrication and repair procedures. The test bench is also standardized and is always ready for quality control. In total, we have gone many times through LYSO-SiPM assembly, and testing procedures and valuable experience has been acquired. Online and offline analyses for the vertical polarization, meaning continuous monitoring of left-right asymmetry are tested and are working reliably. In the second commissioning beam time after polarimeter installation, we have tested in-plane polarization measurement online software based on the timestamp capability of the readout system. The comparison of the new data acquisition hardware-software with old (already tested system) time stamping system shows superior performance to previous detectors (EDDA, WASA) at COSY. The event rate is increased, and the event selection is very precise based on $\triangle \mathrm{E}$ over $\mathrm{E}$ analysis. Currently, we can analyze online more than a million events per second, which exceeds the event recording rate several times. As a summary, we can conclude: the polarimeter is fully functional and is ready to be used during the first-ever storage ring deuteron EDM measurement at COSY. 


\section{Acknowledgment}

The authors wish to acknowledge support of the EDM project the European Research Council via an ERC AdG (srEDM, contract number 694340). We also thank all involved members of the JEDI collaboration for their dedication and persistence towards this long-term project. This work was supported by Shota Rustaveli National Scientific Foundation of Georgia (SRNSFG), grant number DI-18-298 (High-precision polarimetry for charged-particle Electric Dipole Moment (EDM) searches in storage rings).

\section{References}

[1] F. Abusaif et al., Storage Ring to Search for Electric Dipole Moments of Charged Particles. CERN-PBC-REPORT-2019-002, (https://arxiv.org/pdf/1912.07881.pdf).

[2] W. Bernreuther, CP Violation and Baryogenesis, 2002 (https://arxiv.org/abs/hep-ph/0205279).

[3] JEDI collaboration web page http://collaborations.fz-juelich.de/ikp/jedi

[4] R. Maier et al., Nucl. Instrum. Methods Phys. Res., Sect. A 390, 1 (1997).

[5] William M. Morse et al., Phys. Rev. ST Accel. Beams 16, 114001 (2013).

[6] J. Slim et al., Nucl. Instrum. Methods Phys. Res., Sect. A(2016) 116.

[7] D. Eversmann et al., Phys. Rev. Lett.115 (2015) 094801.

[8] G. Guidoboni et al., Phys. Rev. Lett.117 (2016) 054801.

[9] H. Hempelmann et al., Phys. Rev. Lett.119 (2017) 014801.

[10] Y. Satou et al., Phys. Lett. B 549, 307 (2002).

[11] Towards EDM Polarimetry, PoS(PSTP2015)026. DOI: https://pos.sissa.it/243/026

[12] I. Keshelashvili et al., 2017 J. Phys.: Conf. Ser. 928012018 https://doi.org/10.1088/1742-6596/928/1/012018

[13] SensL ArrayJ-30020-64P-PCB http://sensl.com/products/sipmarrays/arrayj/

[14] KETEK PA3315-WB-0808 https://www.ketek.net/sipm/sipm-products/oem-arrays/

[15] O. Javakhishvili at al., arXiv:2002.01700v1 [physics.ins-det] 5 Feb 2020 https://arxiv.org/pdf/2002.01700.pdf

[16] F. Müller et al., 2017 J. Phys.: Conf. Ser.928 012019 https://doi.org/10.1088/1742-6596/928/1/012019

[17] Development of a Rogowski coil Beam Position Monitor for Electric Dipole Moment measurements at storage rings, Ph.D. thesis Fabian Trinkel http://collaborations.fz-juelich.de/ikp/jedi/documents/theses.shtml

[18] Struck SIS3316-250-14 16 channel 250 MSPS 14-bit http://www.struck.de/sis3316.html

[19] Saint-Gobain, Cerium doped Lutetium PreLude420 crystal. http://www.crystals.saint-gobain.com/PreLude_420_Scintillator.aspx 